\title{
Assessing urban heat-related adaptation strategies under multiple futures for a major U.S. city
}

\author{
Guillaume Rohat ${ }^{1,2,3} \cdot$ Olga Wilhelmi $^{1}$ (D) Johannes Flacke ${ }^{3} \cdot$ Andrew Monaghan $^{4}$. \\ Jing $\mathrm{Gao}^{5} \cdot$ Martin van Maarseveen ${ }^{3} \cdot \mathrm{Hy} \mathrm{Dao}^{2}$
}

Received: 26 June 2020 / Accepted: 6 January 2021/Published online: 27February 2021

(C) The Author(s) 2021

\begin{abstract}
Urban areas are increasingly affected by extreme heat in the face of climate change, while the size and vulnerability of exposed populations are shifting due to economic development, demographic change, and urbanization. In addition to the need to assess future urban heat-related health risks, there is also an increasing need to design adaptation strategies that will be effective under varying levels of socioeconomic development and climate change. We use the case study of Houston, Texas, to develop and demonstrate a scenario-based approach to explore the effectiveness of both autonomous and planned heat-related adaptations under multiple plausible futures. We couple a heat risk model with urban climate projections (under the Representative Concentration Pathways) and vulnerability projections (under locally extended Shared Socioeconomic Pathways) to investigate the impact of different adaptation strategies under multiple scenario combinations. We demonstrate that, in the context of Houston, community-based adaptation strategies aiming to reduce social isolation are the most effective and the least challenging to implement across all plausible futures. Scenario-based approaches can provide local policymakers with context-specific assessments of possible adaptation strategies that account for uncertain futures.
\end{abstract}

Keywords Houston $\cdot$ Climate $\cdot$ Adaptation $\cdot$ Heat $\cdot$ Health $\cdot$ Scenarios

Olga Wilhelmi

olgaw@ucar.edu

1 National Center for Atmospheric Research, Boulder, CO, USA

2 Institute for Environmental Sciences, University of Geneva, Geneva, Switzerland

3 Faculty of Geo-Information Science and Earth Observation, University of Twente, Enschede, The Netherlands

4 Research Computing, University of Colorado Boulder, Boulder, CO, USA

5 Department of Geography \& Data Science Institute, University of Delaware, Newark, DE, USA 


\section{Introduction}

Extreme heat is one of the main causes of weather-related mortality worldwide (Hales et al. 2014), particularly in urban areas (Romero-Lankao et al. 2012), where people are concentrated and where the urban heat island (UHI) effect leads to higher inner-city temperatures (Oke 1973). A wide range of studies have shown that climate change increases the intensity, frequency, duration, and spatial extent of extreme heat events (Dosio et al. 2018), population exposure to such events (Jones et al. 2018; Russo et al. 2019), and ultimately heat-related mortality worldwide (Gasparrini et al. 2017). However, not all population groups will be impacted similarly. Research has demonstrated the greater heat-related vulnerability of certain population groups (Wilhelmi and Hayden 2010), such as low-income communities, those without access to air conditioning, the elderly, ethnic minorities, those with pre-existing medical conditions, or socially isolated persons (Bao et al. 2015; Uejio et al. 2011).

Planned adaptation shows great potential to reduce the future health burden of extreme heat, particularly among the most vulnerable groups (Hondula et al. 2015; Larsen 2015; Liotta et al. 2018). Adaptation to climate change is a complex process encompassing a broad range of actions that take many forms, are triggered by different events, have different objectives, operate on different spatial and temporal scales, involve different actors, are associated with different constraints to implementation, and have context-specific effectiveness (Adger et al. 2005; Holman et al. 2019).

Heat-related adaptation strategies are generally classified and categorized based on three major aspects (e.g., Boeckmann and Rohn 2014; Fernandez Milan and Creutzig 2015; Füssel 2007; Hondula et al. 2015; Wolf et al. 2009). First, they differ based on the aspect of heat risk that they target. Adaptation can target (i) the heat hazard, e.g., through urban design to mitigate the UHI, (ii) the vulnerability of individuals exposed to extreme heat, e.g., through community-based programs and outreach, or (iii) the adaptive capacity of the institutions, e.g., through improvements to early warning systems. Second, adaptation strategies are differentiated based on what is required to implement them, with the distinction between "hard" and "soft" adaptation measures. "Hard" measures relate to the built infrastructure and are often costly to implement and have long-term goals. "Soft" adaptation measures are socialand institutional-based measures that necessitate little or no technological actions and are generally easier to implement and more flexible than "hard" adaptation measures. Third, adaptation strategies vary based on the nature of the actors involved in their implementation. They can originate from governments (i.e., at multiple scales and across multiple agencies and departments), businesses, local communities, and local formal or informal institutions (Juhola et al. 2011; Tompkins and Eakin 2012).

The new scenario framework for climate change research (hereafter, SSP-RCP framework) consists of climate change scenarios (Representative Concentration Pathways (RCPs); van Vuuren et al. 2011) and socioeconomic scenarios (Shared Socioeconomic Pathways, SSPs; O'Neill et al. 2017). The publication of this framework led to a growing body of literature that explores how different combinations of climatic and socioeconomic pathways influence future heat-related impacts (e.g., Jones et al. 2018; Marsha et al. 2018; Rohat et al. 2019a, b; Russo et al. 2019). Despite the fact that the SSP-RCP framework offers new perspectives to explore adaptation strategies and their associated costs and benefits (van Vuuren et al. 2014; Wilbanks and Ebi 2014) and that adaptation is considered to be an important aspect in the majority of climate change impacts, adaptation, and vulnerability (IAV) studies (Barnett 2010; Füssel 
2007; Holman et al. 2019), there has been little use of the SSP-RCP framework to inform adaptation planning until recently.

Among the $\sim 30$ heat-related IAV studies using the SSP-RCP framework, only Anderson et al. (2018) considered adaptation (through increases in individual-level adaptability). Adaptation is also rarely investigated in IAV assessments focusing on other climate impacts. A few notable exceptions can be found in flood-related studies (Alfieri et al. 2016; Hinkel et al. 2014; Scussolini et al. 2018; Ward et al. 2017), cross-sectoral and agricultural studies (Ausseil et al. 2019; Hasegawa et al. 2014; Hölscher et al. 2017; Rutledge et al. 2017), and in studies that propose conceptual SSP-RCP frameworks that account for adaptation (Cradock-Henry et al. 2018; Frame et al. 2018; Kebede et al. 2018). Building upon the aforementioned studies, this paper seeks to advance the consideration of adaptation within IAV studies that rely on the SSP-RCP framework. We use the case study of heat-health risk in Houston, Texas, to demonstrate how adaptation can be embedded within the SSP-RCP framework and to demonstrate the ways in which this framework can be used to assess the effectiveness of adaptation strategies under multiple futures. The overarching goals of this paper are to (i) advance methodology for assessing adaptation strategies within the SSP-RCP framework, (ii) demonstrate how this methodology can provide a range of plausible, policy-relevant outcomes for extreme heat adaptation in Houston, Texas, and (iii) highlight the potential effectiveness of different adaptation strategies with regard to heat-health risks.

\subsection{Adaptation within the SSP-RCP framework}

The SSP-RCP framework has been designed to be combined with Shared Policy Assumptions (SPAs) - made of mitigation and adaptation strategies (Kriegler et al. 2014) -into a scenario matrix architecture (van Vuuren et al. 2014). However, their use has been limited so far (Kebede et al. 2018). Integrated in the SSP-RCP framework as a third axis (Fig. 1), the effectiveness of adaptation strategies (represented by the SPAs) can be assessed across all SSPRCP combinations (e.g., Anderson et al. 2018; Hasegawa et al. 2014; Hinkel et al. 2014). Going further, Hölscher et al. (2017) analyzed the consistency between adaptation-oriented SPAs and different SSP-RCP combinations and subsequently assessed qualitatively the effectiveness of different SPAs across their respective set of consistent SSP-RCP

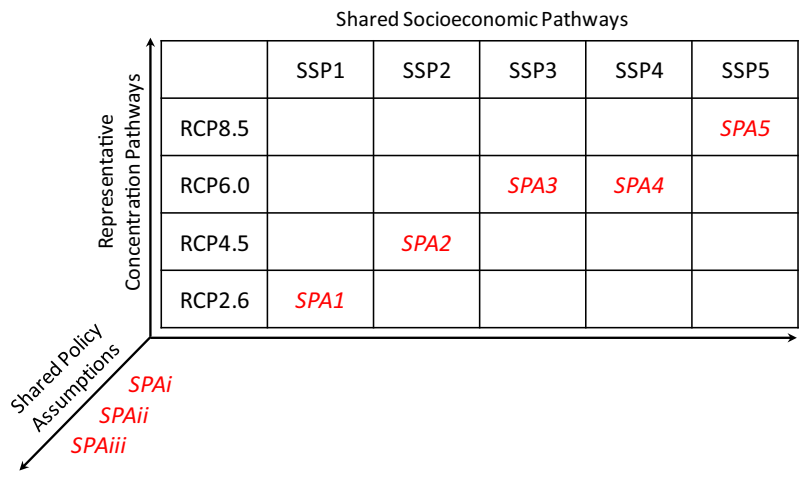

Fig. 1 Integration of adaptation-oriented Shared Policy Assumptions (SPAs) within the scenario matrix architecture, either as a third axis $(S P A i, S P A i i, \ldots)$ to be combined with all (or a selection of) SSP-RCP combinations, or directly embedded within a given SSP-RCP combination $(S P A 1, S P A 2, \ldots)$ to create integrated SSP-RCP-SPA scenarios. Figure adapted from van Vuuren et al. (2014) 
combinations. A few studies also linked a specific adaptation-oriented SPA with a given SSPRCP combination to create integrated SSP-RCP-SPA scenarios (Ausseil et al. 2019; Reimann et al. 2019).

The number of adaptation strategies assessed within a given study varies widely, from one (e.g., Hasegawa et al. 2014) to many (e.g., Hölscher et al. 2017). The complexity and level of detail of the adaptation strategies differ greatly as well, ranging from a straightforwardly implied acclimatization of the population (Anderson et al. 2018) to SPAs made of a combination of several context-specific adaptation strategies (Scussolini et al. 2018). In all IAV studies exploring adaptation under the SSP-RCP framework, the complexity of adaptationwith regard to its different forms, objectives, actors involved, actions required, and constraints - is largely underestimated. This may be due to the poor ability of climate change impact models to represent adaptation (Holman et al. 2019).

It is also crucial to recognize the different degrees of autonomous adaptation assumed under each SSP (autonomous adaptation is not a conscious response to climate change, but rather triggered by socioeconomic development) (Rothman et al. 2014). Globally, SSP1 and SSP5 depict low challenges to adaptation - i.e., high autonomous adaptation - through high education, high health investments, reduced inequality (particularly under SSP1), and effective international cooperation. By contrast, SSP3 and SSP4 depict high challenges to adaptation-i.e., weak autonomous adaptation - due to low or unequal education, low or unequal access to health facilities, water, and sanitation, and high inequality. SSP2 depicts moderate challenges to adaptation. It is worth pointing out that these global assumptions of autonomous adaptation can unfold differently for a particular region and climate-related hazard because adaptation is largely context specific.

\section{Methods}

This study builds on the methodological framework developed in Rohat et al. (2019c) to examine the role of adaptation options in future heat health risks. The overall modeling framework and the incremental advancements in quantifying heat-related adaptation scenarios are shown in Fig. S1. The methodology presented here employs an interdisciplinary approach where the quantitative adaptation scenarios are informed by quantitative and qualitative social science research on extreme heat adaptive capacity (Wilhelmi and Hayden 2016; Hayden et al. 2017).

\subsection{Case study}

The metropolitan area of Houston, Texas, (hereafter Houston) is located in the southeast region of the United States (Fig. 2). It is one of the fastest growing and most diverse urban areas in the USA (Emerson et al. 2012). It currently faces a profound transformation of its social fabric, with growing income inequality, growing racial generation gap (i.e., older white population and increasingly diverse younger population), and expanding urbanization (PolicyLink 2013). Houston's population is also rapidly aging as baby boomers approach their senior years (HCAAA 2016). In addition, Houston faces significant heat-related threats (Papalexiou et al. 2018) which are amplified by climate change (Oleson et al. 2013) and the UHI (Conlon et al. 2016; Zhou et al. 2014). Extreme heat in Houston lead to substantial excess summer mortality among the most vulnerable groups (Chien et al. 2016; Heaton et al. 2014; Zhang et al. 2015). 


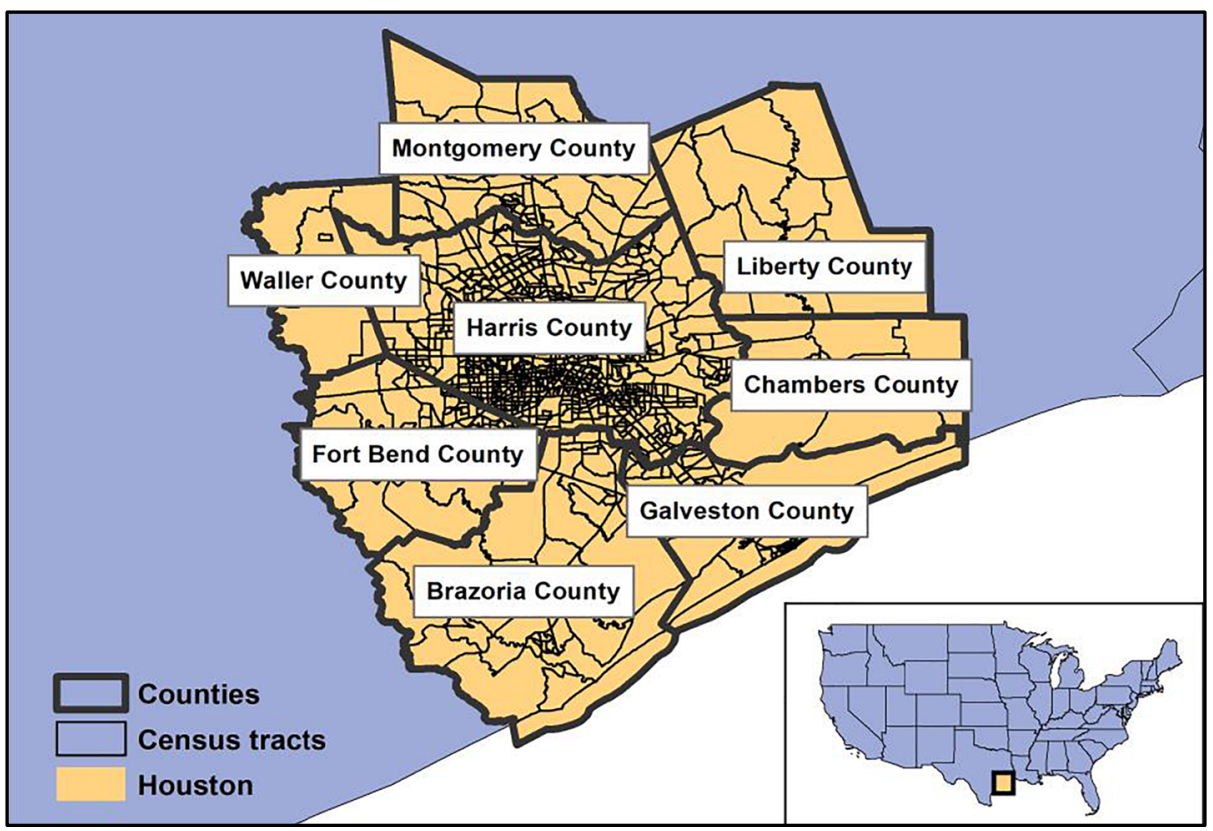

Fig. 2 Spatial extent of Greater Houston (here referred as "Houston") with its 8 different counties and 1600 census tracts (thereafter "tracts")

Houston's high exposure to extreme temperature and its rapidly changing society make it a relevant case study to explore ways in which climate change and socioeconomic development will shape future heat-health risk and to analyze how different adaptation strategies can reduce such risk. We focus on a mid-term time horizon (2050), in line with existing regional development plans in Houston.

\subsection{Heat risk model}

We rely on an existing heat risk model developed for Houston by Heaton et al. (2014). In this hierarchical model, counts of heat-related mortality are modeled using negative binomial regression (to account for overdispersion). While details are provided in Heaton et al. (2014), the basic relative risk equation of the model is

$$
\log \left(R R_{y t b}\right)=\beta_{0}+\text { heat }_{y t b} \beta_{b}+\text { covariates }+\mathrm{SRE}
$$

where $R R_{y t b}$ is the relative risk of a heat-related mortality in year $y$, time $t$, and block group $b$, heat is some measure of heat with block-group specific slope $\beta_{b}$, covariates includes linear effects for various sociodemographic characteristics, and $S R E$ is a spatial random effect capturing spatial correlation between block groups. In this model, the block group specific slopes $\beta_{b}$ are hierarchically modeled and explained by sociodemographics and housing characteristics of each block group in order to understand who is most at risk for increases in heat. The authors found the risk of heat-related summer mortality to be strongly correlated with (i) demographic factors including age and ethnicity; (ii) socioeconomic factors including social isolation, air conditioning prevalence, and poverty; and (iii) climatic determinants and in particular daily minimum temperature as the most strongly correlated heat metric. The latter 
suggests that the inability to cool off at night is an important contributor to risk of heat-related mortality (Kovats and Hajat 2008). Further, Heaton et al. (2014) established that persons older than 65 and living alone are the most at risk for increases in temperature.

In this study, we use only the first layer of the Heaton heat risk model to explore the future relative risk of heat-related mortality. The relative risk of mortality is referred to as "vulnerability" throughout the paper. We assume constant demographic conditions (that is, similar age structure and ethnic diversity as of year 2010) to focus only on the influence of predictive socioeconomic variables for which adaptation strategies can be designed (i.e., social isolation, air conditioning, and poverty). Even though we do not make use of the second layer of the heat risk model, we still examine how different climatic and socioeconomic pathways influence the heat hazard (i.e., daily minimum temperature).

\subsection{Scenario setting}

The SSPs are global development trends and should be extended - that is, contextualized for a specific region and/or sector - to increase their suitability and relevance for local-scale IAV studies (Kriegler et al. 2012; van Ruijven et al. 2014). We used the four extended SSPs for Houston (H-SSPs) developed in Rohat et al. (2019c), which describe alternative development trends for Houston in the 2050s (Table 1 and Text S1).

We employed RCP4.5 and RCP8.5 to account for uncertainty in future greenhouse gas emissions. RCP4.5 is a stabilization scenario that implies a range of climate policies (van Vuuren et al. 2011). By contrast, RCP8.5 depicts continued growth of greenhouse gas emissions throughout the twenty-first century. As some SSP-RCP combinations are unlikely to arise in practice (Kriegler et al. 2012), we focused on a selection of six consistent combinations, namely, H-SSP1*RCP4.5, H-SSP2*RCP4.5, H-SSP2*RCP8.5, H$\mathrm{SSP} 3 * \mathrm{RCP} 8.5, \mathrm{H}-\mathrm{SSP} 5 * \mathrm{RCP} 4.5$, and H-SSP5*RCP8.5.

Table 1 Qualitative development trends of key elements of the four H-SSPs. "++" is high increase, "+" is moderate increase, "=" is static, "-"is moderate decrease, and "- -"is high decrease, relative to the current conditions. Table adapted from Rohat et al. (2019c)

\begin{tabular}{lllll}
\hline Key elements & $\begin{array}{l}\text { H-SSP1: } \\
\text { sustainable } \\
\text { density }\end{array}$ & $\begin{array}{l}\text { H-SSP2: } \\
\text { middle } \\
\text { of the road }\end{array}$ & $\begin{array}{l}\text { H-SSP3: } \\
\text { economic } \\
\text { slowdown }\end{array}$ & $\begin{array}{l}\text { H-SSP5: } \\
\text { pro- } \\
\text { business }\end{array}$ \\
\hline Population growth & + & + & - & ++ \\
Racial/ethnic diversity & ++ & ++ & + & ++ \\
Population aging & + & + & ++ & + \\
Economic growth & + & + & + & + \\
Economic inequalities & -- & + & - & ++ \\
Technology development & ++ & + & + & $=$ \\
Urban vertical development & ++ & + & - & ++ \\
Urban sprawling & -- & - & - & $=$ \\
Societal cohesion & ++ & $=$ & + & $=$ \\
Social policies & ++ & + & - & + \\
Marginalized communities & - & $=$ & - & + \\
Access to affordable air conditioning & ++ & + & & + \\
Access to affordable education & ++ & & & +
\end{tabular}




\subsection{Socioeconomic and land use projections}

This study relied on socioeconomic and land use projections developed by Rohat et al. (2019c) at the census tract level for the year 2050 under the four H-SSPs Specifically, we used (i) the share of single-person households (i.e., social isolation), which were projected using a headship-based approach (McCue and Herbert 2016); (ii) the share of persons in povertythat is, below the poverty thresholds set by the US Census Bureau (2018) - which were projected using a cubic spline correlation model with median income and state-level GDP projections (Absar and Preston 2015; Marsha et al. 2018); and (iii) the share of households without central air conditioning (AC), which were projected using a multiple correlation model fit to projections of household income and building stock (see Rohat et al. (2019c) for more details).

Because urban land use is a critical determinant of Houston's UHI (Conlon et al. 2016), we also retrieved from Rohat et al. (2019c) future land use projections under the four H-SSPs, which were purposely designed to serve as inputs for the urban climate model described next (Fig. S2).

\subsection{Urban climate projections}

Daily minimum temperature (Tmin) in Houston was simulated using an offline version of the Noah land surface model, the High-Resolution Land Data Assimilation System (HRLDAS v3.2; Chen et al. 2007). HRLDAS was coupled with a 1-layer urban canopy model depicting the urban surface to simulate the UHI effect across Houston. HRLDAS was driven at the upper boundary by hourly meteorological forcing data for the period 1991-2010 from Phase 2 of the North American Land Assimilation System (NLDAS-2; Xia et al. 2012).

Using the current and projected urban land use as inputs to HRLDAS, we simulated the baseline (1991-2010) summer daily Tmin across Houston under current and future (H-SSP-specific) land use patterns. To account for the RCP-driven changes in climatic conditions, we employed the computationally efficient process described in Conlon et al. (2016), Marsha et al. (2018), and Rohat et al. (2019c). First, we retrieved daily Tmin for June, July, and August from historical (1991-2010, referred hereafter as the 2000s) and future (2041-2060, referred hereafter as the 2050s) simulations from six regional climate models (RCMs; see Table S1). The future simulations were for the RCP4.5 and RCP8.5 scenarios. Second, we computed daily deltas of Tmin between future and historical time periods for each RCP and each RCM simulation. Third, we employed the statistical downscaling procedure described in Marsha et al. (2018) to adjust the historical HRLDAS simulations according to the RCM projections under RCP4.5 and RCP8.5, using the daily deltas (see Text S2). This process was repeated for each RCM, each RCP, and each HRLDAS historical simulation. Finally, we aggregated the resulting 1-km climate projections to the U.S. census tract scale. This process resulted in a set of tract-level projections of summer daily Tmin across Houston, under different combinations of land use scenarios (baseline and four $\mathrm{H}$ SSPs) and climate scenarios (two RCPs), for the 2050s. We used the multi-model mean to display the results. We also analyzed the climate projections in terms of the number of warm nights per summer, that is, the number of days where Tmin reaches or exceeds $27{ }^{\circ} \mathrm{C}\left(\sim 80^{\circ} \mathrm{F}\right)$ (Rohat et al. 2019c). 


\subsection{Adaptation strategies}

\subsubsection{Design and quantification}

There are numerous existing adaptation strategies to reduce heat-related health risk in urban areas. As noted previously, adaptation strategies differ in the aspect of heat risk they target, what is required to implement them, and the nature of actors involved. To explore existing heat-related adaptation strategies in Houston, we reviewed Houston's development plans, resilience plans, and climate action plans (City of Houston 2018, 2019b; H-GAC 2018; Mayoral Task Force on Equity 2017; WHA 2007, 2018). Although these plans do not explicitly focus on climate adaptation, they refer to heat risk-related fields, such as urban planning, social vulnerability, aging, and marginalized communities. In addition, we reviewed 11 dedicated adaptation plans of North American cities (see Table S2) to explore concrete heat-related adaptation options in the North American context. We also used the results from a Houston household survey on extreme heat vulnerability and adaptive capacity (Hayden et al. 2017) to contextualize heat-related adaptive capacity of the households across Houston. Survey results revealed that (i) among the most common measures taken by Houstonians to protect themselves from extreme heat were staying indoors and using AC, (ii) there is little awareness about the existence of heat-related social programs (e.g., assistance programs to help pay electricity bills and cooling centers), and (iii) social networks are an important aspect of adaptive capacity. Finally, we used the results from a Houston stakeholder survey on extreme heat vulnerability and adaptation (Wilhelmi and Hayden 2016) to characterize the perception of Houston's stakeholders about the effectiveness of existing heat preparedness and response activities and the feasibility of future strategies for heat risk reduction. Results showed that Houston's stakeholders view improvements in preparedness and community-based adaptation as effective measures for reducing heat-related vulnerability.

The review of plans and survey results informed the creation of a list of contextspecific adaptation strategies, from which we used a subset of strategies that specifically target the predictive drivers in heat risk model, i.e., heat hazard, social isolation, poverty, and AC prevalence (Table 2). Quantifying these adaptation strategies was challenging due to the absence quantifiable adaptation goals in the reviewed climate adaptation plans and the lack of empirical evidence on the effect of specific strategies on a given adaptation target. Despite those challenges, we quantified the adaptation strategies as follows:

- Adaptation strategy targeting the heat hazard: we quantified its effect by assuming that the UHI will remain at the historic level (year 2010).

- Adaptation strategies targeting vulnerability: we quantified their effect by assuming that they would lead to a decrease in the maximum tract-level values of their respective target variable (i.e., households without central AC, share of persons living in poverty, or share of persons living alone). We set the maximum values according to the ambition of the adaptation strategy (high or low) and to the current tract-level distribution of the variables' values. Specifically, we set the maximum values at the 50th and 75 th percentiles (rounded to $\pm 5 \%$ ) for high- and low-ambition adaptation strategies respectively. 


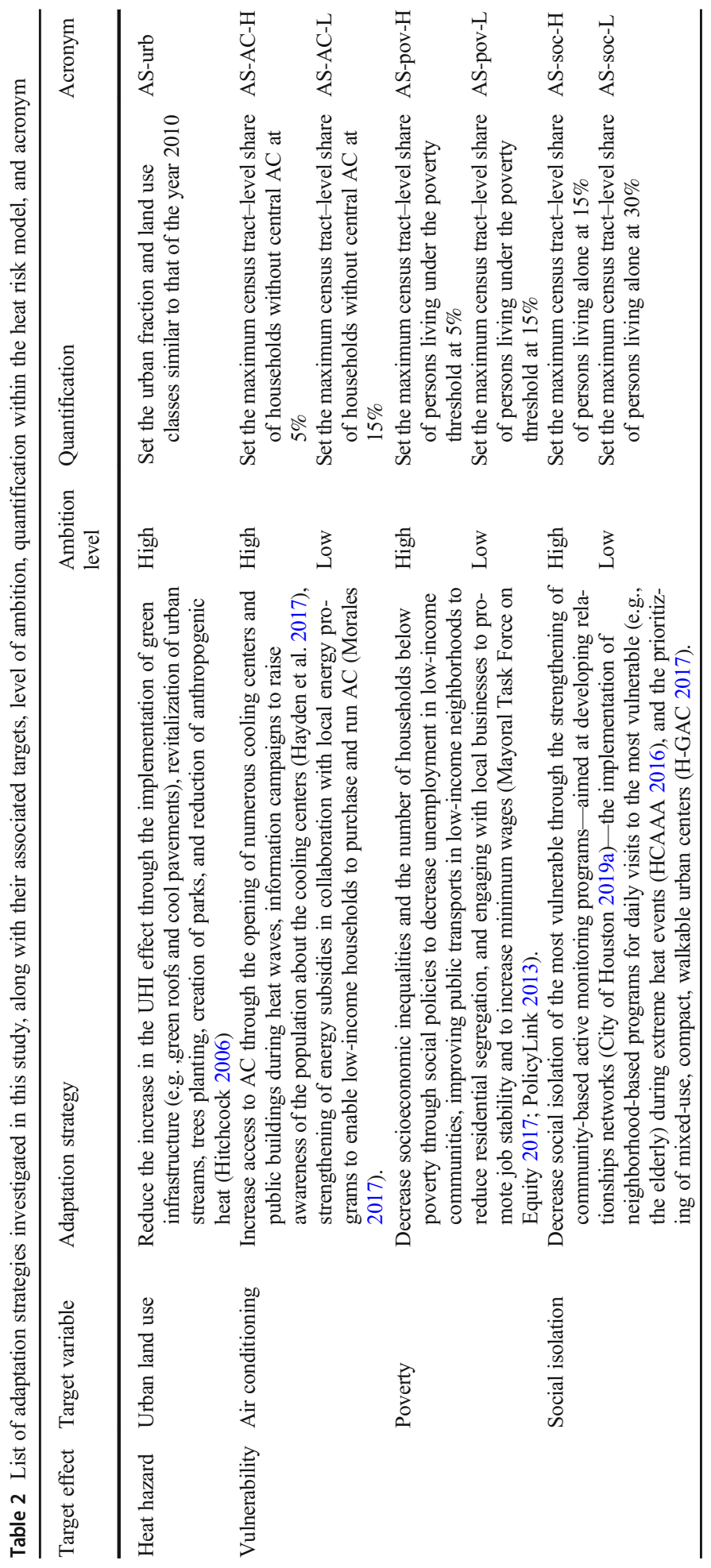




\subsubsection{Assessment of effectiveness}

We used two different approaches to assess the effectiveness of adaptation strategies, depending on their target. For the adaptation strategy targeting the heat hazard (i.e., AS-urb), we considered its effectiveness in terms of its ability to decrease the mean summer daily Tmin and the number of warm nights (at both tract and county levels). In addition, we compared the effectiveness of AS-urb with that of mitigation (i.e., a shift from RCP8.5 to RCP4.5).

For the adaptation strategies targeting vulnerability (i.e., AS-AC, AS-pov, and ASsoc), we created the concept of "adaptation range" to assess their effectiveness in a standardized fashion. We defined the adaptation range as the percentage decrease in vulnerability (at the tract level, relative to the vulnerability in year 2010) when the socioeconomic predictive variables of heat-related vulnerability were set to null (i.e., no poverty, social isolation, or lack of AC). In other words, the adaptation range represents the maximum possible decrease in vulnerability - hence the maximum possible range of adaptation-for each tract. Across Houston, the adaptation range is $\sim 17 \%$ in average (that is, a maximum mean decrease in vulnerability of 17\%), with tract-level values ranging from 1 to $52 \%$ (Fig. S3).

We then considered the effectiveness of a given adaptation strategy as its ability to "fill" the adaptation range, thus expressed in percentage (\%). As an example, if the adaptation range of tracts $\mathrm{A}$ and $\mathrm{B}$ is $20 \%$ and $40 \%$, respectively, and that an adaptation strategy leads to a $5 \%$ decrease in vulnerability in tract $A$ and $10 \%$ in tract B; then, its effectiveness is $25 \%(5 / 20$ and $10 / 40$ ) in both tracts. Such an approach enables exploring the effectiveness of adaptation strategies in a standardized way across Houston's tracts.

\section{Results and discussion}

\subsection{Heat hazard projection}

Results of the urban climate simulations show that daily Tmin is projected to increase across Houston under all scenarios. The mean summer Tmin increases from $24.7{ }^{\circ} \mathrm{C}$ in the $2000 \mathrm{~s}$ to $26.2{ }^{\circ} \mathrm{C}-27.1{ }^{\circ} \mathrm{C}$ (interquartile range $=0.6$ ) in the $2050 \mathrm{~s}$ - depending on the H-SSP*RCP combination. The changes are unevenly distributed across Houston area, with the southern coastal counties showing both the highest temperatures and the largest increases (Fig. S4a).

The number of warm nights $\left(\mathrm{Tmin}>27^{\circ} \mathrm{C}\right.$ ) also increases under all scenarios (Fig. S4a), shifting from less than 1 night per summer in most counties during the 2000s to over 20 nights per summer in most counties during the 2050 s. In half of the counties, the number of warm nights under RCP8.5 reaches over 50 per summer. Spatial patterns of Tmin (Fig. S4b) highlight the substantial influence that the type of urbanization pathway has on the future urban climate, particularly in areas expected to shift from rural to urban. The compact urban development depicted in H-SSP1 leads to a much smaller increase in daily Tmin than the sprawling urban development depicted in H-SSP5. Assuming RCP4.5 climate, the summer mean Tmin is $0.27{ }^{\circ} \mathrm{C}$ greater for H-SSP5 versus H-SSP1 on average across Houston and $0.7^{\circ} \mathrm{C}$ greater innumerous newly urbanized census tracts.

In addition to the urbanization type, the climate scenario is also an important determinant of future urban climatic conditions. The summer mean Tmin is $\sim 0.51{ }^{\circ} \mathrm{C}$ greater for RCP8.5 versus RCP4.5 on average, and can reach $>1{ }^{\circ} \mathrm{C}$ in some tracts. The influence of the climate 
scenario on future temperature conditions is therefore, in most cases, greater than that of the socioeconomic/urbanization scenario for the Houston area.

\subsection{Adaptation targeting the heat hazard}

In addition to projecting the future heat hazard, we assessed the ability of the specific adaptation strategy to reduce the future heat hazard by implementing green infrastructure, planting trees, etc. (see Table 2 "Heat Hazard" target effect), under different H-SSP*RCP combinations. Results show that this adaptation strategy leads to substantial reduction of the heat hazard, but with important differences in effectiveness across socioeconomic scenarios and counties (Fig. 3a).

Under a scenario of a compact and sustainable city (H-SSP1), this adaptation strategy has very little influence (it leads to a decrease $<0.05{ }^{\circ} \mathrm{C}$ in most counties), mainly because of the low increase in the UHI effect depicted under this scenario. By contrast, under a scenario depicting a sprawling city (H-SSP3 and H-SSP5), this adaptation strategy leads to large reduction of the heat hazard, with a mean decrease in daily Tmin of $\sim 0.2-0.3{ }^{\circ} \mathrm{C}$ in newly urbanized counties. Although significant, this adaptation-driven reduction of the heat hazard should be put in perspective with the potential mitigation-driven reduction (i.e., reaching RCP4.5 instead of RCP8.5) of the mean summer Tmin, which attains at least $\sim 0.5-0.6{ }^{\circ} \mathrm{C}$ in most tracts.

We found similar results when using the number of warm nights as the representation of the heat hazard (Fig. 3b). Under scenarios depicting a strong climate change and a sprawling city (H-SSP3*RCP8.5 and H-SSP5*RCP8.5), the adaptation-driven reduction in the number of warm nights can reach more than 7 days per summer on average across a few counties but is limited to a reduction of $<4$ days per summer on average across most counties. In scenarios depicting a compact city (H-SSP1) or a mixed development pattern (H-SSP2), the adaptation-

a

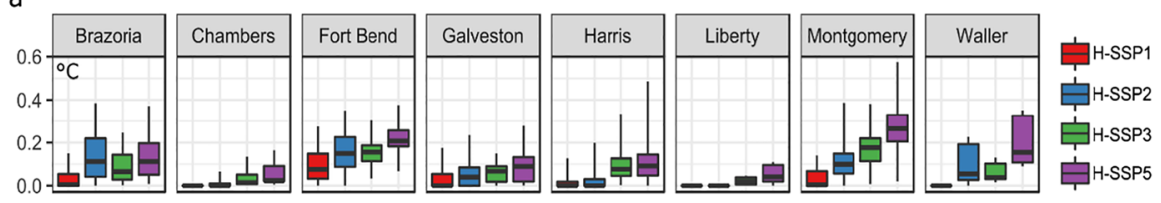

b

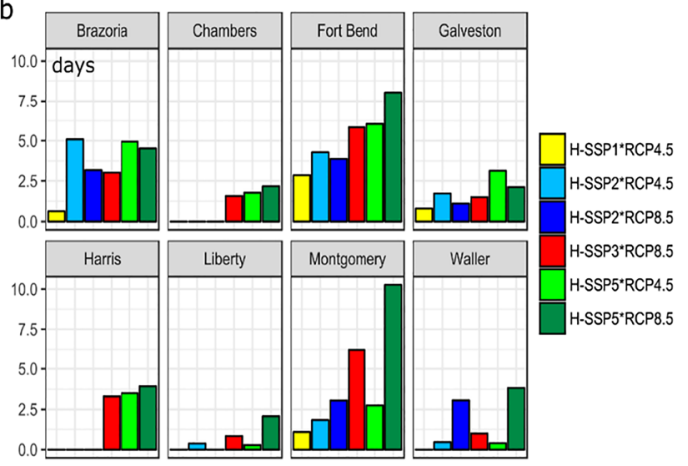

C

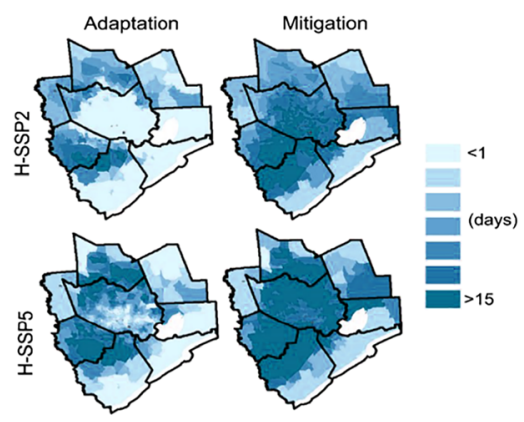

Fig. 3 a Census tract-level distribution of decrease in Tmin $\left({ }^{\circ} \mathrm{C}\right)$ due to adaptation, under each $\mathrm{H}-\mathrm{SSP}$, for each county. b County-level mean decrease in the number of warm nights (days per summer), under each H-SSP*RCP combinations, for each county. c Census tract-level decrease in the number of warm nights (days per summer) due to adaptation or mitigation, for H-SSP2 and H-SSP5 
driven reduction in the number of warm nights is very low on average $(<1$ day per summer, in most cases), even when considering the RCP8.5 climate scenario. The mitigation-driven reduction is higher in most cases (5-20 days per summer on average depending on the county). Similar findings apply at the tract level (Fig. 3c). The adaptation-driven reduction in the number of warm nights is significant on the outskirts of Harris County - particularly under a scenario of a sprawling city (H-SSP5) — but less than the mitigation-driven reduction in most tracts (Fig. 3c).

Overall, for the vast majority of tracts, achieving RCP4.5 instead of RCP8.5 (i.e., mitigation) would lead to a higher reduction in the heat hazard compared to implementing the adaptation strategy investigated here. However, the latter is far from negligible and plays a particularly important role in the reduction of the heat hazard in newly urbanized areas (e.g. Fort Bend, Montgomery, and Waller counties) under scenarios depicting a sprawling city (HSSP3 and H-SSP5).

\subsection{Vulnerability projections and autonomous adaptation}

We first explored projections of vulnerability under each H-SSP without adaptation strategies. County-level results (Fig. 4a) highlight the disparities in future vulnerability across scenarios and counties. H-SSP1 and H-SSP5, depicting both a decrease in socioeconomic inequality, an increase in social capital, and an increase in access to AC, leading to the lowest levels of vulnerability. On the contrary, H-SSP3, depicting an economic slowdown and a decrease in
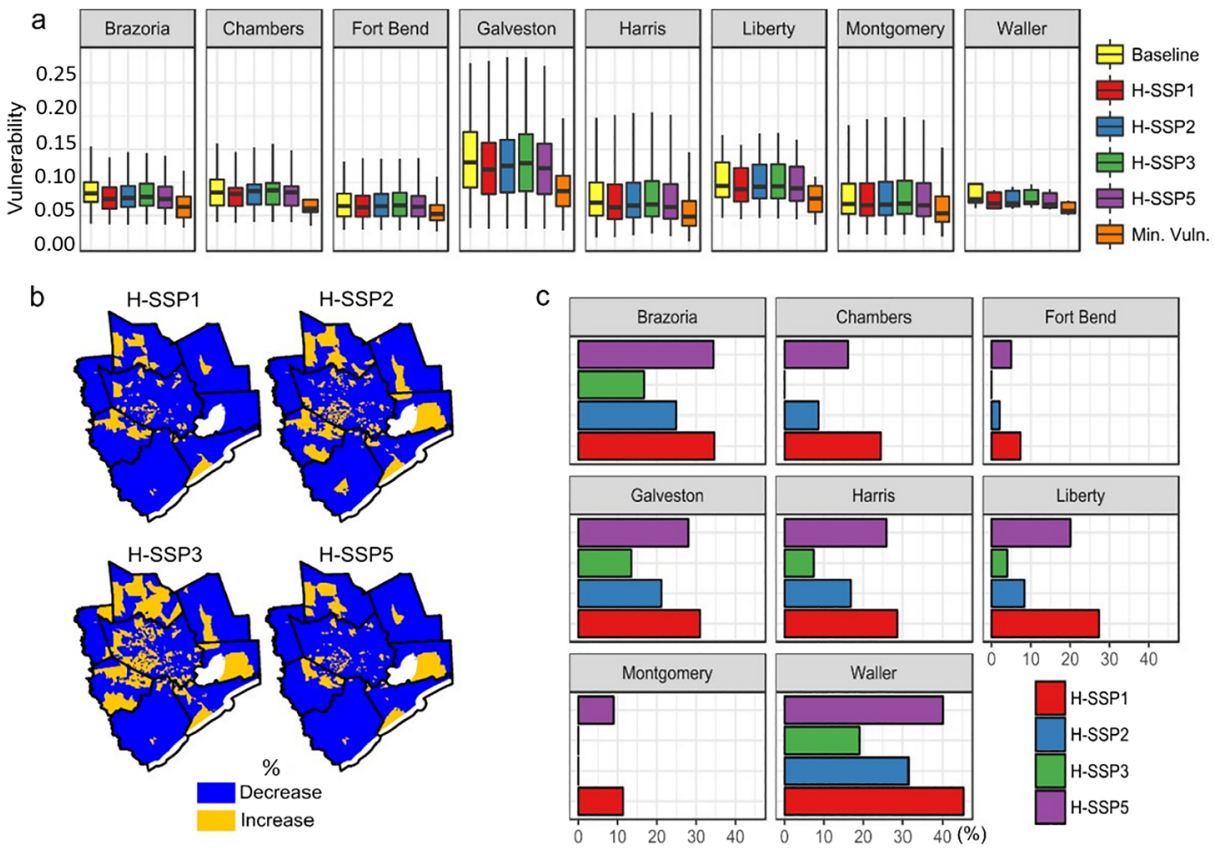

Fig. 4 a Census tract -level distribution of vulnerability under each H-SSP, under baseline conditions (year 2010), and under a scenario of minimum vulnerability (Min. Vuln.) - note that the difference between the baseline and Min. Vuln. represents the adaptation range. b Census tract-level spatial patterns of increase or decrease in vulnerability relative to the baseline, under each H-SSP. c County-level mean effectiveness (in \%) of the autonomous adaptation implied under each H-SSP 
job stability and purchasing power, leads to the highest levels of vulnerability. Large differences remain across tracts and across counties.

Although H-SSP1 and H-SSP5 lead to decreased vulnerability, they do not reach the minimum possible vulnerability (i.e., vulnerability assuming full adaptation) (Fig. 4b). This means that adaptation strategies could still play an important role in decreasing vulnerability under these socioeconomic pathways.

A large proportion of the census tracts that have historically been most vulnerable-located in the center and southeast of Harris County (Heaton et al. 2014; Rohat et al. 2019c) — show a decrease in vulnerability under all scenarios (Fig. 4b), highlighting the autonomous adaptation (gradual adaptation resulting from socioeconomic development) that takes place under all $\mathrm{H}$ SSPs in most counties (Fig. 4c). Aligned with the projections of vulnerability, autonomous adaptation is expected to be greater under H-SSP1 and H-SSP5 than under other scenarios, although relatively low $(<15 \%$ on average) under all scenarios in certain counties such as Montgomery and Fort Bend. This emphasizes the need to augment autonomous adaptation with planned adaptation under all types of socioeconomic development.

\subsection{Adaptation-driven decrease in vulnerability}

We assessed the ability of different adaptation strategies to decrease future vulnerability separately under each H-SSP. Results (Fig. 5a) show that there is no one-size-fits all adaptation strategy (AS) and that the effectiveness of a given AS is highly dependent on (i) the location (here the county) in which it is implemented, (ii) the socioeconomic scenario considered, (iii) its level of ambition, and (iv) its target. Overall, results show that a highly ambitious AS targeting social isolation (AS-soc-H) is the most effective across all counties (effectiveness > $20 \%$ in most counties and reaching $>30 \%$ in Liberty and Montgomery Counties) and under all scenarios. In Harris County, where most of Houston's population is located and where the shares of persons in poverty and of households without AC are the highest, strategies targeting poverty (AS-pov) and prevalence of AC (AS-AC) are the most effective (effectiveness $~ 15-$ $25 \%$ ), particularly under the H-SSP2 and H-SSP3 scenarios. Under the latter scenarios, ASpov and AS-AC have an effectiveness $\sim 2$ to 3 times higher than under H-SSP1 and H-SSP5, highlighting the different effectiveness of a same AS across multiple plausible futures.

We also explored the implementation of combinations of strategies with both low (AS-allL) and high (AS-all-H) ambition. Census tract-level effectiveness exhibits substantial differences across tracts, highlighting the location dependency of effectiveness (Fig. 5b). Both ASall-L and AS-all-H have an effectiveness $>40 \%$ in urban tracts of Harris County, while in most other tracts only AS-all-H is effective. In the same way as the effectiveness of a single AS, the effectiveness of combined strategies varies across scenarios.

It is crucial to point out that the effectiveness of planned adaptation strategies is complemented by the autonomous adaptation depicted under each H-SSP. We compared the mean total adaptation effectiveness (i.e., the sum of autonomous and planned adaptation) across counties, H-SSPs, and ambition of planned adaptation. Results highlight the effectiveness of highly ambitious planned adaptation, relative to that of autonomous adaptation (Fig. 5c). Autonomous adaptation plays a secondary role in most counties and under all scenarios (particularly under H-SSP2 and H-SSP3). This underlines the ability of a highly ambitious planned adaptation strategy to decrease future vulnerability under multiple plausible socioeconomic trajectories, even under those that depict highly vulnerable populations with weak autonomous adaptation. On the contrary, planned adaptation with low ambition will be much 
a
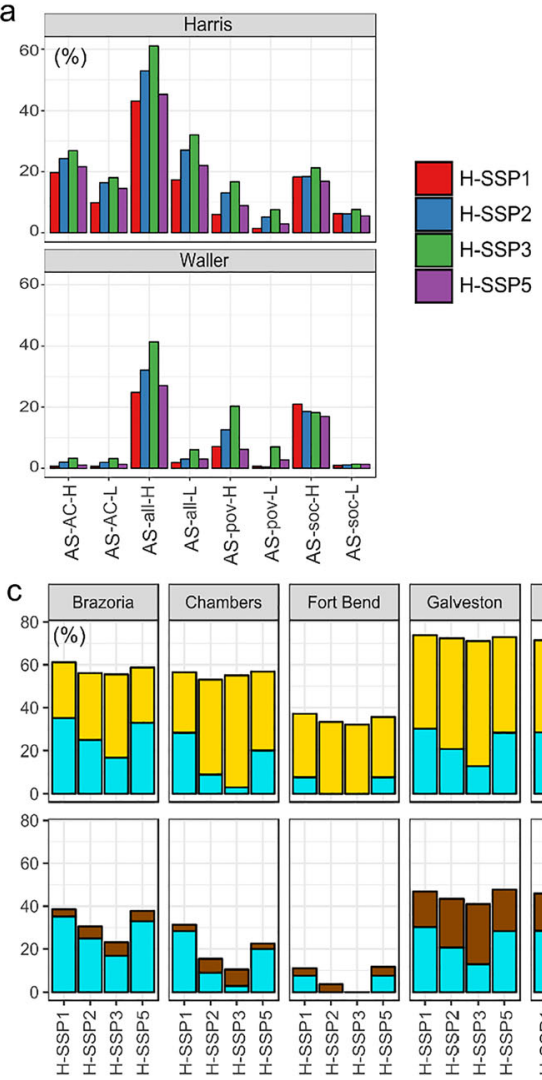

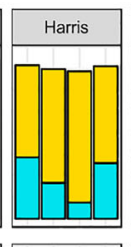

b AS-all-L
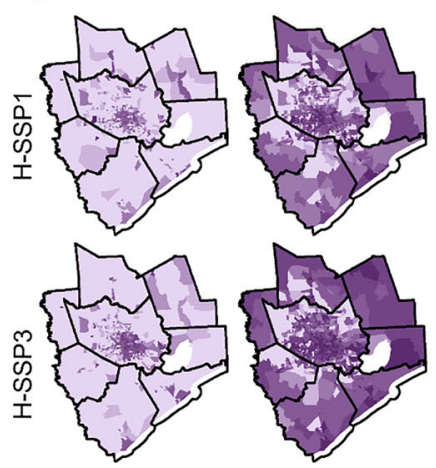
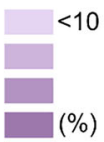

$(\%)$

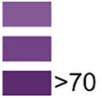

$>70$
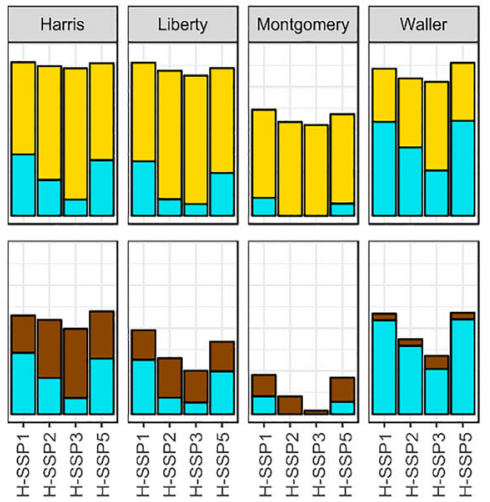

$\square$ AS-all-H

AS-all-L
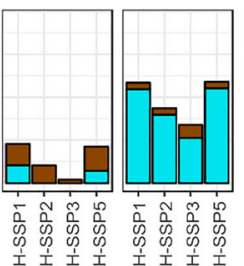

Autonomous

Fig. 5 a County-level mean effectiveness (\%) of adaptation strategies (targeting AC, poverty (pov), or social isolation (soc) and associated with high (H) or low (L) ambition), under each H-SSP, for Harris and Waller Counties. b Census tract-level spatial pattern of effectiveness (\%) of combinations of adaptation strategies under H-SSP1 and H-SSP3. c County-level mean effectiveness (\%) of the addition of autonomous adaptation and combinations of adaptation strategies (AS-all-H or AS-all-L), under each H-SSP. See Table 2 for the adaptation strategies' acronyms

less effective than the spontaneous autonomous adaptation in most counties and under most scenarios.

\subsection{Effectiveness versus challenges to implementation}

In addition to exhibiting different effectiveness (in terms of reduction of heat hazard and/or vulnerability) under the different H-SSPs, the range of adaptation strategies has different $\mathrm{H}$ SSP-specific challenges to implementation. While some adaptation strategies might be highly effective under a given H-SSP, they may also be highly challenging to implement in that given socioeconomic scenario. The ease of implementation largely depends on the technological constraints, political and societal contexts, and economic situation depicted under each H-SSP.

We related the quantitative effectiveness of adaptation strategies (categorized into low, medium, and high effectiveness - see details in Table S3) with their challenges to implementation, under each H-SSP. The resulting matrix-which focuses only on Harris County since we demonstrated that the effectiveness of adaptation strategies largely differs across 
counties - highlights the disparities of both effectiveness and challenges to implementation across scenarios (Fig. 6).

While most strategies present low challenges to implementation under H-SSP1 (because of a large decrease in economic inequality, increased access to affordable AC and increased societal cohesion and social policies), their effectiveness is low-with the exception of highly ambitious strategies targeting the prevalence of $\mathrm{AC}$ and the social isolation. By contrast, most AS show a high effectiveness under H-SSP3 but are associated with high challenges to implementation (e.g. AS-AC-H, AS-urb, and AS-pov-H), due to the comparatively increased economic inequalities, decreased social policies, decreased access to affordable $\mathrm{AC}$, and low technological development depicted under this scenario. Under H-SSP5 — which depicts large increases in technological development and strong economic growth-AS-urb and AS-AC-H are both highly effective and associated with low to medium challenges to implementation.

The main goal of this approach is to pinpoint adaptation strategies that are both effective and least challenging to implement under all plausible futures. In the case of Harris Countywhere most of Houston's population lives - a highly ambitious adaptation strategy targeting social isolation is effective under all scenarios (medium or high effectiveness) and presents low to medium challenges to implementation under all scenarios. This suggests that it is the most feasible and flexible adaptation strategy. A strong increase in the prevalence of AC is also associated with a high effectiveness under all scenarios but would be highly challenging to implement under a scenario of economic slowdown (H-SSP3). It is worth mentioning that because these adaptation strategies are targeting vulnerability, their effectiveness is independent of the climate pathway; only the strategy targeting urbanization (AS-urb) is RCPdependent.

\section{Conclusions}

Using the case study of Houston, Texas, we developed and demonstrated an approach to explore the effectiveness of adaptation strategies under multiple futures. Using a heat risk model (Heaton et al. 2014) in combination with RCP-based urban climate projections and $\mathrm{H}$ SSP-based projections of vulnerability, we assessed the effectiveness of both autonomous and planned adaptation (related to the reduction of the UHI, decrease in poverty, increase in AC

H-SSP1

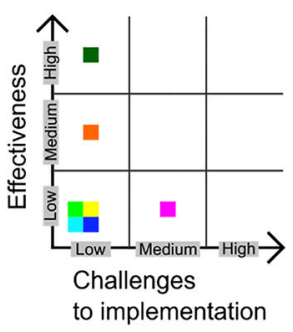

H-SSP2

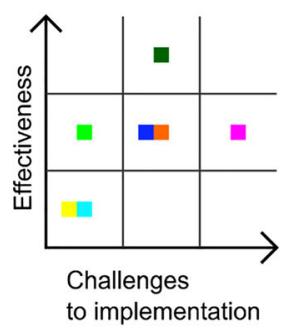

H-SSP3

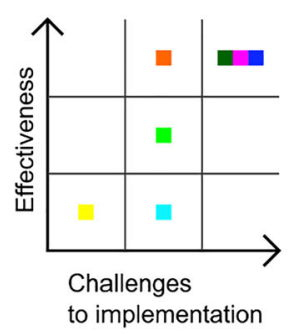

H-SSP5

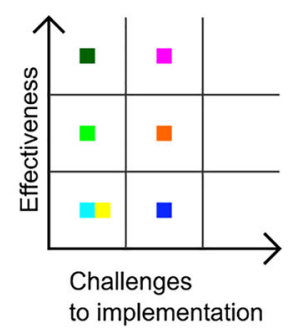

AS-urb aS-AC-L

-AS-AC-H AS-SOC-L

aS-SOc-H $\quad$ AS-pov-L

aS-pov-H

Fig. 6 Effectiveness of adaptation strategies in relation to challenges to implementation (categorized in low, medium, and high), under each H-SSP, for each adaptation strategy individually, with a focus on Harris County for all adaptation strategies except AS-urb (multi-county focus instead). See Table 2 for the adaptation strategies' acronyms 
prevalence, and decrease in social isolation) under each H-SSP. Balancing the effectiveness of each AS with its challenges to implementation, we demonstrated that a highly ambitious adaptation strategy targeting social isolation may be particularly effective for the Houston area (especially for densely populated Harris County) and would be relatively easy-to-implement under most scenarios. This result is consistent with a Houston stakeholder survey (Wilhelmi and Hayden 2016) which showed that community outreach and interventions are effective activities for extreme heat response and strengthening community-based adaptation is an effective pathway to decrease future heat vulnerability.

We also demonstrated that the effectiveness of an adaptation strategy is largely dependent on (i) the aspect of heat risk it targets, (ii) the type of socioeconomic development, (iii) the level of climate change (for strategies targeting the heat hazard), (iv) the communities it targets, and (v) the location where it is implemented. The two latter aspects underscore the need to design adaptation plans that offer diverse set of strategies that allow for context-appropriate implementation decisions. The SSP-RCP framework is a useful tool to explore the effectiveness of adaptation strategies in the context of uncertainty regarding future socioeconomic and climatic trajectories (CradockHenry et al. 2018), particularly when embedded within the scenario matrix as a third axis. This approach is particularly useful for comparing the effectiveness across multiple scenarios. When exploring adaptation strategies under all of the H-SSPs, we found it critical to account for the challenges to implementation under each H-SSP (whereby the challenge is related to the consistency between a given scenario and adaptation strategy), as adaptation is restrained and conditioned by technological and institutional capacities and availability of resources (Berkhout 2012).

Quantitative assessment of adaptation strategies for heat-related risk is constrained by current understanding of the relationships between urban heat hazard, vulnerability, and risk (Wilhelmi and Hayden 2010). In this case study, our ability to explore the effectiveness of adaptation strategies quantitatively was limited by the low number of predictive variables of heat-health risk depicted in the Heaton et al. (2014) model. Future research could investigate adaptation strategies targeting other potential drivers of individual vulnerability (e.g., education and pre-existing medical conditions) and drivers of institutional adaptive capacity (e.g., effectiveness of early warning systems and inter-agency cooperation). Further research would also benefit from the use of heat risk models that integrate a larger number of predictive variables upon which the effectiveness of a wider range of adaptation strategies could be assessed (Inostroza et al. 2016; Wolf and McGregor 2013).

In addition to being constrained by the predictive variables of the heat risk model, our quantitative assessment of adaptation strategies was also largely limited by the absence of quantifiable targets within existing adaptation plans. We were also greatly limited by the lack of empirical evidence on the quantitative and practical effect of governmental policies and community-based programs targeting specific aspects of heat-related vulnerability. Therefore, we used a simplified approach to quantify the influence of different adaptation strategies that underestimates the complexity of adaptation with regard to its different actors involved, actions required, constraints, and time lag of implementation (Holman et al. 2019). Further research to enhance the ability of climate impact models to represent the multi-faceted aspects of adaptation would be highly beneficial. To achieve this, we view stakeholder engagement to be a critical activity (Wilhelmi and Hayden 2016). Local stakeholders are likely to provide valuable context-specific insights on adaptation strategies, particularly concerning (i) their local relevance, (ii) their influence on certain aspects of heat-health risk, and (iii) their associated 
requirements and constraints to implementation under different socioeconomic trajectories. The future work could involve stakeholders' contributions through a participatory modeling approach, and in doing so contextualize the model for a specific location. The wide range of translation methods (Mallampalli et al. 2016) also shows great potential to assess stakeholder insights on the quantitative effect of adaptation strategies on certain aspects of heat-health risk.

Building upon this work, further research could make use of the richness of existing urban climate models to assess the ability of specific "hard" adaptation measures (e.g., green walls, vegetated or white rooftops, and revitalized urban streams) to decrease the UHI and the heat hazard under different climate scenarios. Further research could also make use of the SSP-RCP framework to assess the costs and benefits of adaptation strategies under multiple SSP-RCP combinations (van Vuuren et al. 2014), with particular attention given to SSP-specific costs of implementation of adaptation measures. Finally, further research could explore maladaptation and trade-offs between adaptation and mitigation (Juhola et al. 2016). This is particularly crucial when suggesting an increase in AC prevalence as an adaptation strategy (Salamanca et al. 2014). Although challenging, these research activities are critical to provide local policymakers and practitioners with more comprehensive and robust information about the adaptation strategies that are required to increase urban resilience under uncertain climatic and socioeconomic conditions.

Supplementary Information The online version contains supplementary material available at https://doi.org/ 10.1007/s10584-021-02990-9.

Acknowledgments The authors would like to thank three anonymous reviewers for their thoughtful suggestions. We also would like to thank Dr. Matthew Heaton for his help with the manuscript revision.

Funding This work was partially funded by the State Secretariat for Education, Research, and Innovation (SERI, Switzerland) within the framework of its program "Cotutelles de these" and by the Swiss National Science Foundation's Doc Mobility scholarship. National Center for Atmospheric Research is sponsored by the National Science Foundation.

Data availability Adaptation plans, aggregated Houston survey data, and socioeconomic and urban climate projections are available upon request. Extended SSPs for Houston are available in Supplemental Material.

\section{Compliance with ethical standards}

Conflicts of interest The authors declare no known conflict of interest.

Code availability $\mathrm{R}$ code for Heaton risk model and statistical downscaling are available upon request.

Open Access This article is licensed under a Creative Commons Attribution 4.0 International License, which permits use, sharing, adaptation, distribution and reproduction in any medium or format, as long as you give appropriate credit to the original author(s) and the source, provide a link to the Creative Commons licence, and indicate if changes were made. The images or other third party material in this article are included in the article's Creative Commons licence, unless indicated otherwise in a credit line to the material. If material is not included in the article's Creative Commons licence and your intended use is not permitted by statutory regulation or exceeds the permitted use, you will need to obtain permission directly from the copyright holder. To view a copy of this licence, visit http://creativecommons.org/licenses/by/4.0/. 


\section{References}

Absar SM, Preston BL (2015) Extending the shared socioeconomic pathways for sub-national impacts, adaptation, and vulnerability studies. Glob Environ Chang 33:83-96

Adger NW, Arnell NW, Tompkins EL (2005) Successful adaptation to climate change across scales. Glob Environ Chang 15:77-86

Alfieri L, Feyen L, Di Baldassarre G (2016) Increasing flood risk under climate change: a pan-European assessment of the benefits of four adaptation strategies. Clim Chang 136:507-521

Anderson GB, Oleson KW, Jones B, Peng RD (2018) Projected trends in high-mortality heatwaves under different scenarios of climate, population, and adaptation in 82 US communities. Clim Chang 146:455-470

Ausseil AGE, Daigneault AJ, Frame B, Teixeira EI (2019) Towards an integrated assessment of climate and socio-economic change impacts and implications in New Zealand. Environ Model Softw 119:1-20

Bao J, Li X, Yu C (2015) The construction and validation of the heat vulnerability index, a review. Int J Environ Res Public Health 12:7220-7234

Barnett J (2010) Adapting to climate change: three key challenges for research and policy-an editorial essay. Wires Clim Chang 1:314-317

Berkhout F (2012) Adaptation to climate change by organizations. Wires Clim Chang 3:91-106

Boeckmann M, Rohn I (2014) Is planned adaptation to heat reducing heat-related mortality and illness? A systematic review. BMC Public Health 14:1112

Chen F, Manning KW, LeMone MA et al (2007) Description and evaluation of the characteristics of the NCAR high-resolution land data assimilation system. J Appl Meteorol Clim 46:694-713

Chien LC, Guo Y, Zhang K (2016) Spatiotemporal analysis of heat and heat wave effects on elderly mortality in Texas, 2006-2011. Sci Total Env 562:845-851

City of Houston H (2018) Climate action plan - resources. City of Houston Office of Sustainability, Houston, Texas

City of Houston H (2019a) Complete communities. City of Houston, Houston, Texas

City of Houston H (2019b) Resilient Houston: resilience assessment. City of Houston, Office of Sustainability, Houston, Texas

Conlon K, Monaghan A, Hayden MH, Wilhelmi O (2016) Potential impacts of future warming and land use changes in intra-urban heat exposure in Houston. Texas PloS One 11

Cradock-Henry NA, Frame B et al (2018) Dynamic adaptive pathways in downscaled climate change scenarios. Clim Chang 150:333-341

Dosio A, Mentaschi L, Fischer EM, Wyser K (2018) Extreme heat waves under $1.5^{\circ} \mathrm{C}$ and $2^{\circ} \mathrm{C}$ global warming. Environ Res Lett 13:054006

Emerson MO, Bratter J, Howell J et al (2012) Houston region grows more racially/ethnically diverse with small declines in segregation - a joint report analyzing census data from 1990, 2000, and 2010. Kinder institute for urban research. Rice University, Houston, Texas

Fernandez Milan B, Creutzig F (2015) Reducing urban heat wave risk in the 21st century. Current Opin Env Sust $14: 221-231$

Frame B, Lawrence J, Ausseil A et al (2018) Adapting global shared socioeconomic pathways for national and local scenarios. Clim Risk Manag 21:39-51

Füssel HM (2007) Adaptation planning for climate change: concepts, assessment approaches, and key lessons. Sustain Sci 2:265-275

Gasparrini A, Guo Y, Sera F et al (2017) Projections of temperature-related excess mortality under climate change scenarios. Lancet Planetary Health 1:e360-e367

H-GAC (2017) Livable centers - more choices, great places. Houson-Galveston Area Council, Houston, Texas

H-GAC (2018) 2045 active transportation plan. The Houston-Galveston Area Council (H-GAC), Houston, Texas

Hales S, Kovats S, Lloyd S, Campbell-Lendrum D (2014) Quantitative risk assessment of the effects of climate change on selected causes of death, 2030s and 2050s. WHO Report, World Health Organization, Geneva, Switzerland

Hasegawa T, Fujimori S, Shin Y et al (2014) Climate change impact and adaptation assessment on food consumption utilizing a new scenario framework. Environ Sci Technol 48:438-445

Hayden MH, Wilhelmi OV, Banerjee D et al (2017) Adaptive capacity to extreme heat: results from a household survey in Houston, Texas. Weather Clim Soc 9:787-799

HCAAA (2016) Progress report for Houston and Harris County: 2008-2016 ageing agenda. Harris County Area Ahency on Ageing (HCAAA), Houston, Texas

Heaton MJ, Sain SR, Greasby TA et al (2014) Characterizing urban vulnerability to heat stress using a spatially varying coefficient model. Spat Spatiotemporal Epidemiol 8:23-33

Hinkel J, Lincke D, Vafeidis AT et al (2014) Coastal flood damage and adaptation costs under $21^{\text {st }}$ century sealevel rise. Proc Natl Acad Sci U S A 111:3292-3297 
Hitchcock D (2006) Strategic plan for urban heat island mitigation in the Houston region. Houston Advanced Research Center, Houston, Texas

Holman IP, Brown C, Carter TR et al (2019) Improving the representation of adaptation in climate change impact models. Reg Environ Chang 19:711-721

Hölscher K, Frantzeskaki N, Holman IP et al (2017) Adaptation and mitigation pathways, and synergy mechanisms between them, for the case studies. Deliverable D4.2, IMPRESSIONS Project

Hondula DM, Balling RC, Vanos JK, Georgescu M (2015) Rising temperatures, human health, and the role of adaptation. Curr Clim Change Rep 1:144-154

Inostroza L, Palme M, de la Barrera F (2016) A heat vulnerability index: spatial patterns of exposure, sensitivity and adaptive capacity for Santiago de Chile. PLoS One 11:e0162464

Jones B, Tebaldi C, O'Neill BC et al (2018) Avoiding population exposure to heat-related extremes: demographic change vs climate change. Clim Chang 146:423-437

Juhola S, Keskitalo ECH, Westerhoff L (2011) Understanding the framings of climate change adaptation across multiple scales of governance in Europe. Environ Polit 20:445-463

Juhola S, Glaas E, Linnér B-O, Neset T-S (2016) Redefining maladaptation. Environ Sci Pol 55(135):140

Kebede AS, Nicholls RJ, Allan A et al (2018) Applying the global RCP-SSP-SPA scenario framework at subnational scale: a multi-scale and participatory scenario approach. Sci Total Env 635:659-672

Kovats RS, Hajat S (2008) Heat stress and public health: a critical review. Annu Rev Public Health 29(41):55

Kriegler E, Edmonds J, Hallegatte S et al (2014) A new scenario framework for climate change research: the concept of shared climate policy assumptions. Clim Chang 122:401-414

Kriegler E, O’Neill BC, Hallegatte S et al (2012) The need for and use of socio-economic scenarios for climate change analysis: a new approach based on shared socio-economic pathways. Glob Environ Chang 22:807822

Larsen L (2015) Urban climate and adaptation strategies. Front Ecol Environ 13:486-492

Liotta G, Inzerilli MC, Palombi L et al (2018) Social interventions to prevent heat-related mortality in the older adult in Rome, Italy: a quasi-experimental study. Int J Environ Res Public Health 15

Mallampalli VR, Mavrommati G, Thompson J et al (2016) Methods for translating narrative scenarios into quantitative assessments of land use change. Environ Model Softw 82:7-20

Marsha A, Sain SR, Heaton MJ et al (2018) Influences of climatic and population changes on heat related mortality in Houston, Texas, USA. Clim Chang 146:471-485

Mayoral Task Force on Equity (2017) Rising together: a roadmap to confront inequality in Houston. City of Houston, Houston, Texas

McCue D, Herbert C (2016) Updated household projections, 2015-2035: methodology and results. Working Paper. Harvard Joint Center for Housing Studies, Cambridge, MA

Morales L (2017) Beat the heat program breathes cool air into Houston. CitySavvy, Houston, Texas

O'Neill BC, Kriegler E, Ebi KL et al (2017) The roads ahead: narratives for shared socioeconomic pathways describing world futures in the 21st century. Glob Environ Chang 42:169-180

Oke TR (1973) City size and the urban heat island. Atmos Environ 7:769-779

Oleson KW, Monaghan A, Wilhelmi O et al (2013) Interactions between urbanization, heat stress, and climate change. Clim Chang 129:525-541

Papalexiou SM, AghaKouchak A, Trenberth KE, Foufoula-Georgiou E (2018) Global, regional, and megacity trends in the highest temperature of the year: diagnostics and evidence for accelerating trends. Earths Future 6:71-78

PolicyLink (2013) An equity profile of the Houston-Galveston region. PolicyLink, Oakland, CA

Reimann L, Jones B, Wolff C, Vafeidis AT (2019) Sea-level rise impacts on spatial population change under the SSP-RCP scenario framework. Scenarios Forum 2019, Denver, CO.

Rohat G, Flacke J, Dosio A et al (2019a) Projections of human exposure to dangerous heat in African cities under multiple socioeconomic and climate scenarios. Earths Future 7:528-546

Rohat G, Flacke J, Dosio A et al (2019b) Influence of changes in socioeconomic and climatic conditions on future heat-related health challenges in Europe. Glob Planet Change 172:45-59

Rohat G, Wilhelmi O, Flacke J et al (2019c) Characterizing the role of socioeconomic pathways in shaping future urban heat-related challenges. Sci Total Env 695:133941

Romero-Lankao P, Qin H, Dickinson K (2012) Urban vulnerability to temperature-related hazards: a metaanalysis and meta-knowledge approach. Glob Environ Chang 22:670-683

Rothman DS, Romero-Lankao P, Schweizer VJ, Bee BA (2014) Challenges to adaptation: a fundamental concept for the shared socio-economic pathways and beyond. Clim Chang 122:495-507

Russo S, Sillmann J, Sippel S et al (2019) Half a degree and rapid socioeconomic development matter for heatwave risk. Nat Commun 10:136

Rutledge DT, Ausseil A, Baisden WT (2017) Identifying feedbacks, understanding cumulative impacts and recognising limits: a national integrated assessment. Synthesis report RA3 
Salamanca F, Georgescu M, Mahalov A et al (2014) Anthropogenic heating of the urban environment due to air conditioning. J Geophys Res-Atmos 119:5949-5965

Scussolini P, Tran VT, Diaz-Loaiza A et al (2018) Adaptation to sea level rise: a multidisciplinary analysis for Ho Chi Minh City, Vietnam. Water Resour Res 10:841-857

Tompkins EL, Eakin H (2012) Managing private and public adaptation to climate change. Glob Environ Chang 22:3-11

Census Bureau, 2018. Income and poverty Online at: https://www.census.gov/topics/income-poverty.html ()

Uejio CK, Wilhelmi OV, Golden JS et al (2011) Intra-urban societal vulnerability to extreme heat: the role of heat exposure and the built environment, socioeconomics, and neighborhood stability. Health Place 17:498507

van Ruijven BJ, Levy MA, Agrawal A et al (2014) Enhancing the relevance of shared socioeconomic pathways for climate change impacts, adaptation and vulnerability research. Clim Chang 122:481-494

van Vuuren DP, Edmonds J, Kainuma M et al (2011) The representative concentration pathways: an overview. Clim Chang 109:5-31

van Vuuren DP, Kriegler E, O’Neill BC et al (2014) A new scenario framework for climate change research: scenario matrix architecture. Clim Chang 122:373-386

Ward PJ, Jongman B, Aerts JC, Bates PD, Botzen WJW, Loaiza AD, Hallegatte S, Kind JM, Kwadijk J, Scussolini P, Winsemius H (2017) A global framework for future costs and benefits of river-flood protection in urban areas. Nature Climate Change 7:642-646

WHA (2007) West Houston Plan 2050: Envisioning greater West Houston at mid-century. West Houston Association (WHA), Houston, Texas

WHA (2018) West Houston 2060 Plan. West Houston Association (WHA), Houston, Texas

Wilbanks TJ, Ebi KL (2014) SSPs from an impact and adaptation perspective. Climatic Change 122:473-479

Wilhelmi O, Hayden M (2016) Reducing vulnerability to extreme heat through interdisciplinary research and stakeholder engagement. In: Extreme weather, health, and communities. Interdisciplinary engagement strategies (Steinberg and Sprigg, Eds.): 165-186. Springer Press

Wilhelmi OV, Hayden MH (2010) Connecting people and place: a new framework for reducing urban vulnerability to extreme heat. Environ Res Lett 5:014021. https://doi.org/10.1088/1748-9326/5/1/014021

Wolf T, McGregor G (2013) The development of a heat wave vulnerability index for London, United Kingdom. Weather and Climate Extremes 1:59-68

Wolf J, Lorenzoni I, Few R, Abrahamson V, Raine R (2009) Conceptual and practical barriers to adaptation: vulnerability and responses to heat waves in the UK. in Lorenzoni I, O'Brien KL, Adger WN (eds.) Adapting to Climate Change: Thresholds, Values, Governance. Cambridge University Press, Cambridge, pp 181-196

Xia Y, Mitchell K, Ek M, Cosgrove B, Sheffield J, Luo L, Alonge C, Wei H, Meng J, Livneh B, Duan Q, Lohmann D (2012) Continental-scale water and energy flux analysis and validation for North American Land Data Assimilation System project phase 2 (NLDAS-2): 2. Validation of model-simulated streamflow. Journal of Geophysical Research: Atmospheres 117:n/a-n/a

Zhang K, Chen T-H, Begley C (2015) Impact of the 2011 heat wave on mortality and emergency department visits in Houston, Texas. Environmental Health 14

Zhou W, Ji S, Chen TH, Hou Y, Zhang K (2014) The 2011 heat wave in Greater Houston: Effects of land use on temperature. Environ Res 135:81-87

Publisher's note Springer Nature remains neutral with regard to jurisdictional claims in published maps and institutional affiliations. 